\title{
ORIENTACIONES PEDAGÓGICAS PARA EL ACOMPAÑAMIENTO EDUCATIVO POR DUELO A PERSONAS ADULTAS CON DISCAPACIDAD INTELECTUAL
}

\section{Pedagogical guidelines for educational accompaniment for grieving to adults with intellectual disabilities}

\section{Orientations pédagogiques pour le soutien éducatif pour le deuil par adultes handicapés mentaux}

Pablo Rodríguez Herrero, Agustín de la Herrán Gascón y Dolores IZuzQuiza GASSET

Universidad Autónoma de Madrid. Facultad de Formación de Profesorado y Educación. Departamento de Didáctica y Teoría de la Educación. Campus de Cantoblanco. Ctra. de Colmenar Viejo, km 15,5. 28049 Madrid. Correo-e: rhpablo@botmail.com,agustin.delaherran@uam.es,lola.izuzquiza@uam.es

Fecha de recepción: enero de 2013

Fecha de aceptación definitiva: abril de 2013

Biblid [(1130-3743) 25, 1-2013, 173-189]

\section{RESUMEN}

Se precisa una fundamentación y orientaciones para la actuación en situaciones de duelo con personas adultas con discapacidad intelectual, adecuadas a sus características. En este trabajo se realiza una revisión de literatura pertinente para fundamentar el acompañamiento educativo como una metodología pedagógica de apoyo al duelo cuya urdimbre contiene tres elementos principales: a) La concepción del duelo desde su potencial educativo, b) La prevención de trastornos asociados a duelos complicados y c) La intervención pedagógica preferiblemente desde la 
tutoría, o desde la actuación de educadores o profesionales cercanos a personas con discapacidad intelectual. El modelo de acompañamiento educativo es una propuesta que puede situarse en los modelos humanistas del duelo, al considerar además de las características del proceso de duelo las posibilidades formativas de su elaboración. En las conclusiones del artículo se describen algunas de las ventajas del acompañamiento educativo en adultos con discapacidad intelectual.

Palabras clave: discapacidad intelectual, acompañamiento educativo, duelo, pedagogía.

\section{SUMMARY}

It is needed a foundation and some orientations to act in loss and grief situations with adults with intellectual disabilities. In this article, through a relevant literature review, we based the educational accompaniment as a pedagogic methodology of support with three principal elements: a) The conception of grief from its formative potential, b) The prevention of disorders associated to grief complications, c) The pedagogic intervention preferably from the tutorship or from the actuation of educators or professionals near to intellectual disabilities people. The educational accompaniment model is a proposal that can be placed on humanist models of grief since it considers both the characteristics of the grieving process and the formative possibilities of its elaboration. The article's conclusions present some of the benefits of the educational accompaniment for adults with intellectual disabilities.

Key words: intellectual disability, educational accompaniment, grief, pedagogy.

\section{SOMMAIRE}

On a besoin d'une fondation et orientations pour l'action dan des situations de deuil avec des adultes ayant une déficience intellectuelle, adaptés à leurs caractéristiques. Ce travail, à travers une revue de la littérature pertinente, est basé soutien éducatif en tant que méthodologie pédagogique pour soutenir le deuil dont la chaîne contient trois éléments principaux: a) Le concept de deuil de leur potentiel éducatif, b) La prévention des troubles liés à un deuil compliqué, et c) l'intervention pédagogique préférence mentorat, ou l'exécution d'éducateurs professionnels ou à proximité de personnes ayant une déficience intellectuelle. Le modèle soutien à l'éducation est une proposition qui peut être placé sur des modèles humains de chagrin, de considérer également les caractéristiques du processus de deuil des possibilités éducatives de leur développement. Les conclusions de l'article décrit certain des avantages d'un soutien éducatif pour les adultes handicapés mentaux.

Mots clés: handicapés mentaux, soutien éducatif, duel, pédagogie. 


\section{INTRODUCCIÓN}

La comunidad profesional y científica ha aceptado -al menos parcialmente- en las últimas décadas que las personas con discapacidad intelectual experimentan también la vivencia del dolor y el proceso de duelo por el fallecimiento de personas significativas (Dodd, Dowling y Hollins, 2005). Este progreso no es suficiente pero asocia implicaciones pedagógicas relevantes para la formación de personas adultas con discapacidad intelectual. Ésta se define, siguiendo a la American Association on Intellectual and Develpmental Disabilities (AAIDD), como una discapacidad caracterizada por limitaciones significativas en el funcionamiento intelectual y la conducta adaptativa manifestada en habilidades prácticas, sociales y conceptuales. Además, esta discapacidad comienza antes de los 18 años (Schalock y col., 2010). Si se acepta el duelo en aquellas personas que, por sus limitaciones intelectuales y desajustes en la conducta adaptativa, pueden tener más dificultades en su elaboración, parece lógico y adecuado el establecimiento de unas bases que fundamenten el acompañamiento educativo por la pérdida de un ser querido.

El acompañamiento educativo (Herrán y Cortina, 2008a) hace referencia a una metodología no directiva de trabajo con educandos de cualquier edad en situaciones de duelo. Empleamos el término "educandos" al considerar que cualquier persona se forma permanentemente -o puede hacerlo- a lo largo de toda la vida. Si bien en el presente artículo se proponen orientaciones para el acompañamiento de personas adultas con discapacidad intelectual, es una metodología que podría emplearse también con esta población desde la edad infantil, ya que pretende ayudar a elaborar el duelo desde la orientación y preferentemente desde la tutoría personalizada (Zabalza y Cid, 2006; Monreal Gimeno y Gordillo, 2009). Cuando hablamos del tutor o tutora como figura de referencia nos estamos refiriendo también, en personas adultas con discapacidad intelectual, a profesionales cercanos como educadores, mediadores laborales, etc. Desde el modelo de calidad de vida centrado en la persona (Schalock y Verdugo, 2002) consistiría en aquel profesional que, por su cercanía y significatividad, pudiera coordinar los apoyos que se le dan a la persona con discapacidad intelectual que ha sufrido la pérdida de un ser querido para el desarrollo en todas las dimensiones de calidad de vida (Schalock y Verdugo, 2002, 2007): bienestar emocional, relaciones interpersonales, bienestar material, desarrollo personal, bienestar físico, autodeterminación, inclusión social y derechos.

La adjetivación de "educativo" que hacemos del término "acompañamiento" no es casual ni gratuita. Al decirlo establecemos unas finalidades que le son propias, otorgando al duelo una cualidad de mejora potencial. Se concibe la pérdida, desde este enfoque, como un proceso doloroso pero que en su devenir puede generar mayor madurez personal (Zacarés y Serra, 1997). Pese a las dificultades que puedan tener algunas personas con discapacidad intelectual en comprender la pérdida, consideramos que creer en sus capacidades y evaluar los apoyos que pueden necesitar para la elaboración del duelo podría facilitar la intervención de los profesionales, preferentemente educadores y tutores, en este acompañamiento educativo. 


\section{EL DUELO COMO POSIBILIDAD EDUCATIVA}

La Real Academia de la Lengua Española encuentra dos acepciones del término duelo en su vigésimo segunda edición (DRAE, 2001). Define aquella relacionada con la pérdida como: a) Dolor, lástima, aflicción o sentimiento; b) Demostraciones que se hacen para manifestar el sentimiento que se tiene por la muerte de alguien; y c) Reunión de parientes, amigos o invitados que asisten a la casa mortuoria, a la conducción del cadáver al cementerio, o a los funerales.

La gran mayoría de autores identifica tres fases del proceso de duelo (Tizón, 2004): 1) Fase de impacto o impasibilidad; 2) Fase de depresión o repliegue; 3) Fase de recuperación, curación o restitución. En nuestra opinión, no solo curación sino posible formación y crecimiento, debido a que la persona no vuelve al estado anterior a la pérdida.

Se han definido numerosos modelos del duelo, algunos de los cuales explicamos a continuación siguiendo la clasificación de Tizón (2004, adaptado):

\section{CuAdro 1. MODElos Del DUelo}

\begin{tabular}{|l|l|}
\hline \multicolumn{1}{|c|}{ Modelo } & \multicolumn{1}{c|}{ Autores } \\
\hline Modelos psicodinámicos & S. Freud, H. Deutsch, M. Klein, R. Spitz, A. Freud, etc. \\
\hline Modelos psicosociales & $\begin{array}{l}\text { E. Lindemann, E. Kübler-Ross, G. Pollock, J. Bowbly, A. } \\
\text { Furman, Dsm-IV, G. Caplan, etc. }\end{array}$ \\
\hline Modelos cognitivos & J. W. Worden, M. P. Cleiren, T. Rando, G. Hagman, etc. \\
\hline Modelos mixtos bio-psico-sociales & J. Bowlby, E. Erikson, D. Meltzer, J. L. Tizón, etc. \\
\hline $\begin{array}{l}\text { Modelos humanistas (categoría añadida a } \\
\text { la clasificación de Tizón, 2004) }\end{array}$ & $\begin{array}{l}\text { V. E. Frankl, A. Carmelo, D. Liberman, R. Bayes, R. Bild, } \\
\text { A. de la Herrán, M. Cortina, etc. }\end{array}$ \\
\hline
\end{tabular}

Fuente: Tizón (2004, adaptado).

La mayoría de los modelos del duelo se han centrado en los procesos que lo constituyen. Otros, especialmente aquellos que denominamos humanistas, contemplan además de los procesos las posibilidades formativas de la elaboración sana, autónoma y completa de la pérdida, cuya construcción de significados asociados y que le dan sentido puede generar mayor madurez y formación. Así: «el trabajo educativo desde una vivencia de muerte, además de ayudar a disolver el sufrimiento y a elaborar el duelo, puede incrementar la complejidad y evolución de la conciencia" (Herrán y Cortina, 2008a, 174).

El acompañamiento educativo por duelo es una metodología complementaria a la normalización de la muerte en la educación desde una lectura e intervención pedagógica previa. El enfoque previo de la Educación para la Muerte (Herrán y cols., 2000) es el que tiene lugar antes de que la muerte golpee. Se fundamenta en sus posibilidades didácticas y formativas. En primer lugar se conceptúa como un objeto de conocimiento y un ámbito curricular de características especiales. 
En efecto, su presencia en la naturaleza y en la realidad diríase que no se corresponde en absoluto con su persistente condición de tabú social y educativo. Como reflejo de ello, la muerte atraviesa áreas, materias y temas de carácter transversal del currículo, sin expresarse. Su ausencia formal en los currícula y en los proyectos educativos o pedagógicos de las diferentes etapas es de larga data. Aunque no dispongamos de espacio para detenernos en esta cuestión, consideramos relevante recordar que Comenio (1984) no incluyó la muerte en su Didáctica Magna como ámbito directamente enseñable en los diferentes grados de escuela. Sin embargo, a través del recurso a la naturaleza estaba presente, sobre todo como parte del ciclo vital. En segundo lugar, el enfoque previo de Educación para la Muerte no se ha de considerar desde una orientación didáctica defensiva, sino claramente formativa. Más concretamente, tiene como desembocadura la complejidad y el desarrollo de la conciencia, desarrollable en todos los niveles educativos de modos adecuados. En síntesis, lo que consideramos enfoque previo de la Educación para la Muerte -que retomamos como base de nuestro estudio- promueve la inclusión curricular de la muerte desde la comunicación didáctica y desde la tutoría a lo largo de todos los niveles educativos, con el fin de potenciar una formación de los alumnos con base en una mayor conciencia de su propia realidad finita y pedagógicamente orientada en la vida que les rodea y en la suya propia (Herrán y cols., 2000; Herrán y Cortina, 2006, 2008b). En los últimos años se han realizado propuestas y estudios científicos en relación a esta aproximación previa, tanto en población sin discapacidad (Rodríguez Herrero, Herrán y Cortina, en prensa) como en el ámbito de la educación de personas con discapacidad intelectual (Rodríguez Herrero, Herrán e Izuzquiza, en prensa).

La propuesta de acompañamiento educativo se sitúa en los modelos humanistas del duelo. Son modelos que encajan mejor con una propuesta pedagógica de acompañamiento al contemplar no solo los procesos imbrincados en el duelo sino también las implicaciones formativas de su elaboración.

Como veremos más adelante, las personas con discapacidad intelectual pueden tener más dificultades a la hora de elaborar el duelo y, por lo tanto, de obtener un sentido educativo. Sin embargo, consideramos que el apoyo del tutor/a o del profesional cercano, como figura de referencia emocional y formativa para el individuo que ha sufrido la pérdida de una persona significativa, puede favorecer este proceso de construcción, reparación y elaboración del duelo. La población objeto de este acompañamiento, por tanto, no es quien sufre un duelo patológico y que puede precisar de una intervención clínica sino cualquier educando que, por haber sufrido la muerte de una persona cercana, se encuentre en unos momentos delicados y difíciles, desde la interiorización del sentido pedagógico del acompañamiento. Por ello suscribimos la reflexión de M. Almendro: "Es sumamente atrayente el que un día se deje de hacer terapia para hacer pedagogía” (1995, 241). 


\section{ACOMPAÑAMIENTO EDUCATIVO, PREVENCIÓN DEL DUELO PATOLÓGICO Y DISCAPACIDAD INTELECTUAL}

La esperanza de vida en las personas con discapacidad intelectual ha aumentado considerablemente en las últimas décadas debido a los avances técnicos y también al proceso de aceptación, integración e inclusión en la sociedad de esta población. En los años 20 del pasado siglo la esperanza de vida en personas con síndrome de Down era de 10 años (Watchman, 2005). Actualmente se sitúa entre 55 y 60 años (Todd, 2002; Watchman, 2005). Este aumento en la esperanza de vida en personas con discapacidad intelectual conlleva que, al ser más largo y rico su ciclo vital, maduren y experimenten, como cualquier otra persona, cambios, pérdidas o fallecimientos de amigos y familiares. Además, se estima que un 40\% de la población con discapacidad intelectual vive con padres que tienen una edad avanzada, y que el 10\% convive con uno de los dos padres de más de setenta años (Watson y Harker, 1993). Este hecho hace que las personas con discapacidad intelectual sean más vulnerables a pérdidas secundarias como el cambio del hogar tras el fallecimiento de un familiar cercano.

La cantidad y calidad de investigaciones acerca del duelo en personas con discapacidad intelectual, sin embargo, no ha concurrido paralelamente a este aumento en la esperanza y calidad de vida, siendo todavía un tema tabú tanto en la investigación en el ámbito de la discapacidad intelectual (Todd, 2002; Dodd, Dowling y Hollins, 2005) como en su atención psicológica y pedagógica. Otro factor que ha podido influir en esta falta de inquietud epistemológica y profesional es la idea de que las personas con discapacidad intelectual no pueden, por sus limitaciones cognoscitivas y relacionales, tener un duelo comparable a las personas sin discapacidad ante el fallecimiento de un ser querido, ni comprender la muerte ajena o la muerte propia. Sin embargo, en los últimos tiempos sí parece aceptado que las personas con discapacidad intelectual sufren también la experiencia de la pérdida de seres queridos y pueden tener problemas adicionales en su elaboración por las limitaciones cognoscitivas o dificultades en la competencia comunicativa (Brickell y Munir, 2008).

De las investigaciones realizadas hasta el momento en este ámbito de estudio destacan aquellas que tratan de encontrar cuáles son los síntomas del duelo patológico. Debido a las dificultades en la comunicación y en la expresión emocional en personas con discapacidad intelectual en ocasiones nos encontramos con que delimitar un duelo normal o elaborado de otro patológico o anormal se convierte en un proceso arduo y complicado. La distinción de un duelo normal con otro que pueda ser patológico, se convierte en un paso previo a la intervención pedagógica que se haga con el individuo.

Dicho de otro modo: entendemos que el acompañamiento educativo por duelo ha de formularse como un proceso formativo por el cual se atienden las necesidades educativas, en su más amplia concepción, de aquella persona que ha sufrido una pérdida significativa. El trabajo empírico que describe 9 casos de 
acompañamiento educativo con niños o adolescentes que figuran en Herrán y Cortina (2008a) nos lleva a concluir que esta metodología es realizable por tutores o profesionales preparados. La mayor parte de las situaciones de duelo se recorren y elaboran satisfactoriamente con esta modalidad de acompañamiento desde una tutoría personalizada. Son excepcionales las situaciones de duelo patológico o anormal en las que el acompañamiento del tutor no es suficiente y hay que recurrir a un especialista del ámbito clínico, que posiblemente tendrá que abordar el caso con una perspectiva sistémica de familia. Creemos que las conclusiones de este estudio pueden extrapolarse a niños, jóvenes y adultos con discapacidad intelectual.

El DSM-IV define lo que otros autores denominan duelo patológico, anormal, etc., como un episodio depresivo mayor. Sin embargo, se comparte actualmente la concepción de que el duelo patológico es un trastorno diferente a la depresión. Existe un consenso sobre los criterios para definir el duelo traumático o patológico (Prigerson y cols., 1999):

\section{Criterio A:}

1. La persona ha experimentado la muerte de alguien muy significativo para ella.

2. La respuesta contiene tres de los siguientes cuatro síntomas:

a. Pensamientos obsesivos sobre el fallecido.

b. Añoranza de la persona fallecida.

c. Búsqueda del fallecido.

d. Sentirse solo como resultado de la muerte.

\section{Criterio B:}

1. Sin objetivos, sentimientos de futilidad sobre el futuro.

2. Sentimiento subjetivo de aturdimiento o desconcierto, desapego, ausencia de respuesta emocional.

3. Dificultad para admitir la muerte.

4. Sentimiento de que la vida esté vacía o carente de sentido.

5. Sensación de que ha muerto una parte de uno mismo.

6. Visión destrozada del mundo.

7. Asume los síntomas e incluso conductas peligrosas de la persona fallecida, o relacionadas con ella de algún modo.

8. Exceso de irritabilidad, amargura o rabia relacionadas con la muerte.

\section{Criterio C:}

Duración de la alteración de al menos dos meses.

\section{Criterio D:}

El trastorno ocasiona un deterioro clínicamente importante en las áreas social, laboral u otras importantes para el funcionamiento de la persona. 
Desde nuestra experiencia, para que indicadores como los anteriores puedan ser bien interpretados y aplicados, es importante contextuarlos en el educando y su situación. En nuestra opinión, en personas con discapacidad intelectual los 3 o 4 primeros meses después de la pérdida no debieran implicar una valoración rigurosa de los criterios de duelo patológico anteriormente expuestos. A partir de ese tiempo se podría pensar en derivar a una ayuda especializada. Pero ese apoyo no debería consistir en llevar al educando a un especialista que le ayude por el fallecimiento de su ser querido. Es el sistema familiar quien debiera compartir las orientaciones pertinentes. El educando solo no es quien suele producir un duelo patológico. Es la familia y el contexto, cómo lo gestiona y su influencia en su hijo/a lo que lo genera: su causa suele ser sistémica. Por ello, en caso de derivarse a un especialista, debería verse a toda la familia. Esto es lo más apropiado y también lo más eficaz.

La distinción entre duelo normal y patológico nos invita a reflexionar sobre el valor del acompañamiento educativo por duelo como actuación preventiva del duelo patológico, considerándose además el valor formativo de su elaboración para la madurez y el crecimiento del individuo. De hecho, nos parece muy relevante reparar en que un tratamiento inadecuado $-\mathrm{O}$ la inexistencia del mismopodría generar en un duelo patológico en personas con discapacidad intelectual (Dodd, Dowling y Hollins, 2005).

Tanto el tutor, el educador o el profesional de referencia como el psicólogo clínico deben conocer los síntomas que definen el duelo patológico en personas con discapacidad intelectual, ya que por sus características tienen un riesgo mayor de no elaborar la pérdida y desarrollar un duelo anormal, patológico o atípico (Brickell y Munir, 2008). Hollins y Esterhuyzen (1997) llevaron a cabo un estudio sistemático sobre las reacciones a la pérdida de un ser querido en 50 personas con discapacidad intelectual. De los participantes, 21 mostraron en el Psychopathology Instrument for Mentally Retarded Adults (PIMRA) síntomas patológicos en al menos una de las subescalas de afectividad, ansiedad o desorden adaptativo. Los autores se encuentran con la dificultad de delimitar lo que sería un duelo normal de uno patológico al no poder concluir que los síntomas no son propios de un duelo que está siendo elaborado. En una investigación que continúa la anterior desde una perspectiva longitudinal (Bonell-Pascual y cols., 1999) se mostró que en 18 de los 21 sujetos que habían tenido síntomas patológicos posteriores al duelo éstos habían desaparecido, utilizando también el PIMRA. Dodd y cols. (2008) realizan una investigación para encontrar los síntomas del duelo complicado en personas con discapacidad intelectual. Un tercio de las personas estudiadas manifiestan síntomas de duelo patológico posterior a la pérdida de un ser querido.

La prevención del duelo patológico irá paralela, a través del acompañamiento educativo, al desarrollo del individuo y su elaboración de la pérdida. Blackman (2003) argumenta que para prevenir la complicación del duelo habrá que considerar:

- $\quad$ La posibilidad de que no se entienda el concepto de muerte. 
- La falta de confianza a la hora de tratar el tema en los profesionales.

- La necesidad de estimar los signos del duelo desde un enfoque longitudinal.

- La relevancia de los apoyos de la comunidad en el duelo.

Nuestra orientación se articula, en cualquier caso, desde la Pedagogía y para la formación, más allá de la intervención clínica paliativa que, por otra parte, podría ser adecuada en situaciones de duelo patológico. Ambos enfoques se diferencian en algunos aspectos esenciales, que se exponen a continuación:

I. Enfoque clínico o tradicional de la intervención por duelo:

- Paciente individual como individuo objeto de la intervención.

- El objetivo es la prevención o curación del duelo patológico.

- Intervienen profesionales especializados y clínicos.

II. Enfoque pedagógico del acompañamiento educativo por duelo:

- Cualquier educando como persona susceptible de acompañar.

- El objetivo es la formación.

- Intervienen profesionales con (auto)formación pedagógica.

La presentación anterior puede aclarar las principales diferencias entre la intervención clínica y el acompañamiento pedagógico o educativo por duelo, siendo conscientes de que no se trata de compartimentarlas, sino de definir el mejor modo de atender una necesidad. O sea, acompañar del modo idóneo al educando o, en su caso, al trabajador, que tiene un profesional de apoyo de referencia. Ambas concepciones son complementarias y quizá las dos deban mirar la realidad holística del fenómeno y la atención integral y centrada en la persona con discapacidad intelectual.

\section{El ACOMPAÑAMIENTO EN EL DUELO DE PERSONAS CON DISCAPACIDAD INTELECTUAL}

La Educación para la Muerte o la Didáctica de la Muerte (Herrán y cols., 2000) -que incluye tres enfoques: previo-preventivo, posterior-paliativo y complejo-evolutivo- no está presente, siquiera desde su lectura posterior-paliativa, en la formación de profesionales de la enseñanza, la Pedagogía o la Psicología. En un estudio realizado por MacHale, McEvoy y Tierney (2009) se indaga en las percepciones que tienen los profesionales de la discapacidad intelectual sobre el duelo en esta población. Los participantes en el estudio se mostraban inquietos y dubitativos acerca de cómo llevar a cabo el acompañamiento o el apoyo en la elaboración del duelo. Por tanto, urge incluir temas perennes o radicales como pueda ser la muerte, en la formación de profesionales de la educación por la certeza de su encuentro en su carrera profesional y por su relevancia para la formación de las personas. Transferimos esta reflexión, que es también una petición, al campo de todos los profesionales de la discapacidad intelectual. 
Si bien escasas son las propuestas de normalización de la muerte como elemento educativo en la formación de niños, jóvenes y adultos con discapacidad intelectual desde un enfoque previo-preventivo (anterior a la ocurrencia de la pérdida), encontramos una mayor cantidad de sugerencias y estudios desde la perspectiva paliativa o posterior a la pérdida.

FEAPS Madrid (2001) presenta desde un enfoque global intervenciones que integran tanto actividades preventivas como paliativas. Desde esta última aproximación - por tanto desde una actuación reactiva o paliativa-, FEAPS propone ofrecer espacios adecuados para la elaboración del duelo, facilitar la información que el educando precise, fomentar la expresión de sus sentimientos, abordar los casos centrándose en la persona, hacer partícipe al individuo con discapacidad intelectual así como sea posible y minimizar las pérdidas secundarias o cambios ocasionados por el fallecimiento de un familiar cercano.

Algunas propuestas se derivan de investigaciones que demuestran la efectividad de programas de acompañamiento. Dowling y cols. (2006) realizan una investigación comparativa sobre los efectos de la intervención con un grupo de personas en duelo en relación a la ausencia de intervención con otro grupo de control. El grupo experimental, que además de una intervención clínica tenía el apoyo derivado de la formación de los profesionales y familiares que les acompañaban, elaboraba un duelo más formativo que los integrantes del grupo de control, mejorando su calidad de vida y salud mental.

Elaborar el duelo por la pérdida de un ser querido implica en cierta forma dar un significado a tal pérdida e integrarlo en la propia personalidad. Es un trabajo que incluye, si así lo podemos denominar, una re-construcción del sujeto perdido, dándole un espacio que en cierta manera sustituya su presencia física. Van Dyke (2003) destaca en este sentido el ámbito espiritual de la elaboración del duelo con personas con discapacidad intelectual. En este apoyo integral a aquel que ha sufrido la pérdida de una persona significativa y que, por su discapacidad, puede mostrar mayores dificultades en su comprensión, se pueden utilizar recursos como fotografías, libros y fotos que suplementen la comunicación verbal (Dodd, Dowling y Hollins, 2005). Con el objetivo de ayudar a los profesionales en este acompañamiento Read (2005) escribe una guía para el duelo con personas con discapacidad intelectual en la que propone actividades con libros de historias, fotografías, creación literaria o la incorporación de estas personas a los rituales funerarios. Otro recurso interesante para profesionales es la guía que elabora Markell (2005) para adecuar los rituales funerarios y simbólicos a la persona con discapacidad intelectual y trabajar el ámbito espiritual del duelo.

Una fase crucial en el acompañamiento son los días posteriores a la pérdida de la persona significativa. Clark (2008) propone presentar la información de forma clara y sencilla, adaptada a las dificultades cognoscitivas. Añade la autora que habrá que cuidar también la ruptura que una muerte provoca en las rutinas de personas que presentan discapacidad intelectual. 
La muerte de un familiar o una persona significativa para el individuo que cursa discapacidad intelectual suele acarrear pérdidas secundarias que pueden causar una desadaptación al medio y también una mayor dificultad en la elaboración del duelo. No es inhabitual que una persona con discapacidad intelectual que ha sufrido la pérdida de un familiar cercano se mude 4 o 5 veces al año (Oswin, 1991). Según Brickell y Munir (2008) las pérdidas secundarias, junto con las limitaciones cognitivas y dificultades comunicativas, son los aspectos más reseñables a la hora de pronosticar una posible complicación en la elaboración del duelo en personas con discapacidad intelectual. Otros autores añaden la inteligencia emocional como variable relevante en la elaboración del duelo (McEvoy, Reid y Guerin, 2002). Se hace pertinente una educación emocional que favorezca en los niños, jóvenes y adultos con discapacidad intelectual el reconocimiento, la aceptación y el control emocional también en situaciones asociadas a la pérdida y el duelo.

\section{ORIENTACIONES PARA EL ACOMPAÑAMIENTO EDUCATIVO CON PERSONAS CON DISCAPACIDAD INTELECTUAL}

La American Association on Mental Retardation -actualmente AAIDD- define en el año 2002 las siguientes dimensiones de apoyo a personas con discapacidad intelectual (Izuzquiza y Cerrillo, 2010): a) capacidades intelectuales; b) conducta adaptativa; c) participación, interacciones y roles sociales; d) salud; y e) contexto. A continuación se presenta una serie de posibles apoyos en el proceso de duelo en cada una de las dimensiones citadas, considerando en todo caso que son orientaciones de carácter general y que habrían de adaptarse a la particularidad de cada persona.

I. Capacidades Intelectuales: Comprensión del concepto de muerte; Información clara, sencilla y adaptada; Utilización de imágenes o historias; Favorecer la expresión emocional.

II. Conducta Adaptativa: Explicación de los rituales funerarios; Adaptación a cambios y nuevas rutinas; Explicación del proceso de duelo; Favorecer la participación en rituales; Explicar que en ocasiones es normal exteriorizar las emociones (en algunos casos nos encontramos con que haber interiorizado normas sociales asociadas al control emocional de forma rigurosa y estricta puede dificultar la expresión emocional y sentimental en situaciones de duelo).

III. Participación, interacción y roles sociales: Minimización de pérdidas secundarias (cambios bruscos en la vida cotidiana derivados de la muerte de una persona cercana de la que se depende en algunas áreas de apoyo); Animar, sin forzar, a hablar de la pérdida; Incluirle en el duelo compartido con otros; Apoyar en la comunicación interpersonal; Favorecer la elaboración emocional de la pérdida en contextos naturales; Apoyar a la familia en la normalización del tema de la muerte y la pérdida en el ambiente familiar, de forma que la persona con discapacidad intelectual pueda compartir más fácilmente sus sentimientos y preocupaciones; 
Si la persona que ha sufrido la pérdida ejerciese una actividad laboral, establecer cauces de comunicación entre la figura de apoyo y los compañeros de trabajo de la persona con discapacidad intelectual, para ofrecer orientaciones sobre cómo normalizar la situación en el entorno laboral.

$I V$. Salud: Identificar síntomas de duelo patológico, una vez transcurridos 3 o 4 meses; Derivar, en su caso, a especialistas clínicos; Orientar a la familia e informar de los síntomas físicos que se suelen dar en el proceso de duelo; Intervenir con compañeros, relaciones de amistad o pareja; Explicar a quien ha tenido la pérdida, si fuese necesario, los síntomas físicos que una persona puede tener a lo largo del proceso de duelo.

$V$. Contexto: Animar la expresión de sentimientos de culpa; Respuesta coherente de la institución de apoyo (consensuada y fruto del trabajo coordinado en el que participe todo el equipo profesional); Planificar el traspaso de información entre profesionales y compañeros de la persona que ha sufrido la pérdida, de forma que un ambiente normalizado impida el surgimiento de rumores, sospechas, etc.; Flexibilizar y adaptar el plan formativo a las necesidades que plantee la situación de duelo; Identificar un profesional o tutor de referencia, que acompañe a quien ha sufrido la pérdida durante el proceso de duelo; Considerar posibles trastornos de salud mental que puedan dificultar la elaboración de la pérdida.

El dolor por la pérdida de una persona significativa es transversal a las dimensiones de apoyo descritas por la AAIDD, de forma que los posibles apoyos habrían de realizarse abarcando cada una de las dimensiones. Estos apoyos tienen un carácter general y no tienen por qué ser válidos y adecuados para todas las personas que cursan discapacidad intelectual. Por el contrario, el acompañamiento en el duelo habría de plantearse desde una intervención centrada en la persona, en sus características y necesidades. Recordamos asimismo que el enfoque que planteamos es desde la posibilidad formativa o educativa del duelo y, por lo tanto, no como una respuesta a un duelo patológico, sino como un acompañamiento a toda persona que ha sufrido una pérdida en aras del desarrollo de capacidades resilientes, de la madurez y de la conducta adaptativa.

En cuanto a las orientaciones comunicativas del tutor o figura de referencia para el desarrollo del acompañamiento educativo como metodología de apoyo formativo a la persona en duelo (Herrán y Cortina, 2008a) pueden ser adecuadas en casos de población adulta con discapacidad intelectual:

- Abrir canales para la expresión posible del educando y respetarla (Valls, 2005). Es preciso, en este sentido, escuchar la voz de personas con discapacidad, evaluar e intervenir desde una perspectiva fenomenológica.

- Permitir que se apoye en nosotros cuando lo necesite para, en cualquier momento, reemprender la escalada.

- $\quad$ Seguirle desde atrás. 
- "Quitarnos de en medio" y retirar esquemas predeterminados, muletillas, etc., para que sean sus necesidades las que van a ser atendidas en primer término desde una empatía distanciada, y no las nuestras.

- Favorecer su autonomía.

- Ofrecer la contención y el apoyo necesario permitiendo que recorra por sí mismo y por entero la tristeza, ya que nuestra experiencia nos informa que una vez se sale de ese túnel la persona queda fortalecida.

- Mantenerse disponible, de modo que nuestra oferta de ayuda no se limite en el tiempo, ya que se dan recaídas (efectos Guadiana) por vivencias, aniversarios, etc.

- Evitar errores habituales, que asimilamos a lo que R. Bild (en Herrán y Cortina, 2008a) llama "las seis reglas fundamentales": No mentir, no interrogar, no juzgar, no imponer, no evadirse y no hacer interpretaciones.

\section{CONClusiones}

La muerte está presente a lo largo del ciclo vital de las personas que cursan discapacidad intelectual, y también está presente de un modo variable en su conciencia (Rodríguez Herrero, Herrán e Izuzquiza, en prensa). La Pedagogía -y concretamente la formación pedagógica del profesional de la educación o del mediador laboral- tiene un gran desafío ante sí, previa superación del tabú asociado. El acompañamiento educativo es un enfoque emergente de gran interés y relevancia para la formación y la práctica pedagógica del tutor, el mediador laboral o la figura educativa de referencia, como profesional de la orientación didáctica con el que la persona a quien se acompaña tiene el mayor vínculo educativo. Precisa de una formación basada en la madurez y la mejora personal, que comprende su formación como profesional reflexivo como condición necesaria pero insuficiente (Herrán, 2011). Se precisa la incorporación de constructos emergentes como la conciencia (Herrán, 1998; Torre, 2006), que a su vez trae temas de profundidad como la muerte muy relacionados con la formación. La práctica de la Educación para la Muerte con personas con discapacidad intelectual es investigable desde perspectivas renovadoras desde una Pedagogía de la Muerte y desarrollable desde el ámbito de la tutoría personalizada. Teniendo en cuenta el recorrido que la Pedagogía de la Muerte lleva en España (Rodríguez Herrero, Herrán y Cortina, en prensa), quizá contemos ya con suficientes fundamentos y orientaciones como para avanzar científicamente en este sentido, tanto desde el quehacer del profesional como desde la investigación educativa.

Desde el punto de vista del modo de llevar a cabo las orientaciones desarrolladas a lo largo del trabajo, entendemos que los modelos de apoyo (Schalock y cols., 2010) y de calidad de vida (Schalock y Verdugo, 2002, 2007) aplicados a la formación de personas con discapacidad intelectual nos ofrecen un marco teórico útil para llevar a cabo la práctica del acompañamiento educativo en situaciones de duelo. Así, siguiendo la propuesta de evaluación del apoyo y 
proceso de planificación de la AAIDD (Schalock y cols., 2010), el acompañamiento podría llevarse a cabo en las siguientes fases flexibles:

a) Identificación de las áreas de apoyo relevantes en la persona que ha sufrido la pérdida a través de una reunión del equipo de profesionales en la que se determine la figura principal-mediador laboral, educador, tutor, etc.- que va a coordinar los apoyos. Siendo el profesional de referencia, no ha de acaparar todos los apoyos sino facilitar que desde entornos naturales, con un enfoque ecológico, la persona que ha sufrido la pérdida pueda elaborarla con normalidad y con el soporte de personas emocionalmente cercanas. Así, la figura de referencia no trabaja tan solo con la persona con discapacidad intelectual, sino también con todo su grupo natural de apoyos (familia, pareja, compañeros de trabajo, amigos...).

b) Identificación de actividades de apoyo relevantes para cada dimensión, habiéndose descrito en el apartado anterior algunas orientaciones en cada una de ellas. En cualquier caso, es sumamente relevante que en el acompañamiento por duelo los apoyos se personalicen a las necesidades del individuo, de forma que se respete cómo vive la propia persona el proceso de elaboración de la pérdida. Esta fase puede contar con la reunión de la figura de referencia con el grupo de apoyo natural de la persona, como pueden ser padres, amigos o pareja, siguiendo la metodología de planificación centrada en la persona (Van Loon, 2009), para orientar y determinar los apoyos que se le van a dar en cada área.

c) Evaluación del nivel o intensidad de las necesidades de apoyo en cada dimensión. Los apoyos, siguiendo el modelo de la AAIDD (Schalock y cols., 2010), pueden ser intermitentes, limitados, extensos y generalizados, con una intensidad distinta. Es decir, es importante valorar si en el acompañamiento la persona necesita un apoyo limitado a un momento determinado, como por ejemplo ante una pérdida secundaria, o más continuado, facilitándole espacios en los que expresarse y recursos para poder hacerlo con mayor facilidad.

d) Establecimiento y aplicación del plan de apoyos individualizado coordinado por el profesional de referencia, realizando un seguimiento del proceso de elaboración de la pérdida y valorando si dicho desarrollo está contribuyendo a su formación, manifestada en una mayor madurez personal.

El enfoque pedagógico del duelo que se ha expuesto en el artículo contempla al tutor o al mediador laboral como profesional de referencia, que a su vez requiere de una gran coordinación entre todo el equipo profesional y la intervención sistémica por los agentes e instituciones que pueden apoyar a la persona con discapacidad intelectual en su proceso de duelo. Es un modelo que no sustituye la posible intervención clínica que haya que realizar en aquellos casos en los que, 
por determinadas circunstancias, deriven en un duelo patológico. Sí se considera, sin embargo, su potencial valor para la prevención de dichas complicaciones.

Es preciso recordar, por último, la importancia de que la Educación para la Muerte no contemple tan solo acciones pedagógicas desde su enfoque paliativo o posterior a la pérdida, sino que se normalicen contenidos relacionados con la pérdida, la muerte o el duelo en la formación antes de la ocurrencia de un fallecimiento cercano o de alguna persona significativa (Rodríguez Herrero, 2012). Es una respuesta educativa necesaria y normalizadora, considerando no solo la perennidad de la muerte para el ser humano, sino también su potencial para la formación, como ya expresara el renacentista M. de Montaigne (1533-1592): "Aquel que enseñare a los hombres a morir, enseñaríales a vivir» $(2003,130)$.

\section{REFERENCIAS BIBLIOGRÁFICAS}

Almendro, M. (1995) Psicología y psicoterapia transpersonal. Barcelona, Kairós.

Blackman, N. (2003) Loss and learning disability. London, Worth Publishing Ltd.

Bonell-Pascual, E.; Huline-Dickens, S.; Hollins, S.; Esterhuyzen, A.; Sedgwick, A.; AbDelNOOR, A. y HuBERT, J. (1999) Bereavement and grief in adults with learning disabilities. A follow-up study. British Journal of Psychiatry, 175, 348-351.

BRICKELL, C. y MuniR, K. (2008) El duelo y sus complicaciones en las personas con discapacidad intelectual. Revista Sindrome de Down, 25, 68-76.

Clark, L. (2008) Cómo ayudar a una persona con síndrome de Down a afrontar la enfermedad terminal y la muerte de un ser querido. Revista Sindrome de Down, 25, 126-131.

COMENIO (1984) Didáctica magna. Madrid, Akal.

DodD, P.; Dowling, S. y Hollins, S. (2005) A review of the emotional, psychiatric and behavioural responses to bereavement in people with intellectual disabilities. Journal of Intellectual Disability Research, 49 (7), 537-543.

Dodd, P.; Guerin, S.; McEvoy, J.; Buckley, S.; Tyrell, J. y Hillery, J. (2008) A study of complicated grief symptoms in people with intellectual disabilities. Journal of Intellectual Disability Research, 52 (5), 415-425.

Dowling, S.; Hubert, J.; White, S. y Hollins, S. (2006) Bereaved adults with intellectual disabilities: a combined randomized controlled trial and qualitative study of two community-based interventions. Journal of Intellectual Disability Research, 50 (4), 277-287.

DRAE (2001) 22. edición. Consultado el 28 de junio de 2010. http://www.rae.es/.

FEAPS (2001) Buenas prácticas en situaciones de duelo en personas con retraso mental. Madrid, Colección FEAPS Madrid.

Herrán, A. de la (1998) La conciencia humana. Hacia una educación transpersonal. Madrid, San Pablo.

Herrán, A. de la y Cortina, M. (2006) La muerte y su didáctica. Manual para Educación Infantil, Primaria y Secundaria. Madrid, Editorial Universitas.

- (2008a) La Práctica del "Acompañamiento Educativo" desde la Tutoría en Situaciones de Duelo. Tendencias Pedagógicas, 13, 157-173.

- (2008b) La educación para la muerte como ámbito formativo: más allá del duelo. Psicooncología, 5 (2-3), 409-424. 
Herrán, A. de la; González, I.; Navarro, M. J.; Bravo, S. y Freire, V. (2000) ¿Todos los Caracoles se Mueren Siempre? Cómo tratar la Muerte en Educación Infantil. Madrid, De la Torre.

Hollins, S. y EsterhuYzen, A. (1997) Bereavement and grief in adults with learning disabilities. British Journal of Psychiatry, 170, 497-501.

IzuzQuiza, D. y Cerrillo, M. R. (2010) Discapacidad intelectual, en De Miguel, S. y CerRILLO, M. R. (coords.) Formación para la inclusión laboral de personas con discapacidad intelectual. Madrid, Pirámide.

MacHale, R.; McEvoy, J. y Tierney, E. (2009) Caregiver perceptions of the understanding of death and need for bereavement support in adults with intellectual disabilities. Journal of Applied Research in Intellectual Disabilities, 22 (6), 574-581.

MARKELL, M. (2005) Helping people with developmental disabilities mourn: practical rituals for caregivers. Fort Collins, CO, Companion Press.

McEvoy, J.; ReID, Y. y GueRIN, S. (2002) Emotion recognition and concept of death in people with learning disabilities. The British Journal of Developmental Disabilities, 48 (2), 83-89.

Monreal Gimeno, M. C. y Gordillo, M. C. (2009) La función tutorial, elemento clave en la reforma de la enseñanza universitaria: Una experiencia en Ciencias Ambientales. Bordón. Revista de Educación, 61 (2), 153-163.

Montaigne, M. de (2003) Ensayos. Barcelona, Ediciones Cátedra.

Oswin, M. (1991) Am I allowed to cry? A study of bereavement amongst people who have learning difficulties. London, Human Horizons.

Prigerson, H.; Shear, M. K.; Jacobs, S.; Reynolds, C.; Maciejewsini, P.; Davidson, J.; Rosenheck, R.; Pilkonis, P.; Wortman, C.; Williams, J.; Widiger, T.; Frank, E.; Kupfer, D. y ZIsoOK, S. (1999) Consensus criteria for traumatic grief. A preliminary empirical test. The British Journal of Psychiatry, 174, 67-73.

READ, S. (2005) Loss, bereavement, and learning disabilities: providing a continuum of support. Learning Disability Practice, 8 (1), 31-37.

Rodríguez Herrero, P. (2012) Pedagogía de la Muerte en personas con discapacidad intelectual. Elaboración, aplicación y evaluación de un programa educativo. Tesis doctoral, Universidad Autónoma de Madrid.

Rodríguez Herrero, P.; Herrán, A. de la y Cortina, M. (2012) Antecedentes de la Pedagogía de la muerte en España. Enseñanza \& Teaching, 30, 175-195.

Rodríguez Herrero, P.; Herrán, A. de la e IzuzQuiza, D. (en prensa) "Y si me muero... ¿dónde está mi futuro?’. Hacia una educación para la muerte en personas con discapacidad intelectual. Educación XX1.

Schalock, R.; Borthwick-Duffy, S.; Bradley, V.; Buntinx, W.; Coulter, D.; Craig, E.; Gómez, S.; Lachapelle, Y.; Luckasson, R.; Reeve, A.; Shogren, K.; Snell, M.; Spreat, S.; Tassé, M.; Thompson, J.; Verdugo, M. A.; Wehmeyer, M. y Yeager, M. (2010) Intellectual disability: Definition, classification, and systems of supports. 11th Edition of the AAIDD Definition Manual. Washington, American Association on Intellectual and Developmental Disabilities.

SCHAlOCK, R. y VeRdugO, M. Á. (2002) The concept of quality of life in buman services: A handbook for human service practitioners. Washington, DC, American Association on Mental Retardation. 
- (2007) El concepto de calidad de vida en los servicios y apoyos para personas con discapacidad intelectual. Siglo Cero. Revista Española sobre Discapacidad Intelectual, 38 (224), 21-36.

Tizón, J. L. (2004) Pérdida, pena, duelo. Vivencias, investigación y asistencia. Barcelona, Fundació Vidal i Barraquer.

TodD, S. (2002) Death does not become us: the absence of death and dying in intellectual disability research. Journal of Gerontological Research, 38 (1-2), 225-239.

Torre, S. de la (2006) Creatividad en educación, en DE LA TORRE, S. y Violant, V. (coords.) Comprender y evaluar la creatividad, vol. I. Málaga, Aljibe, 123-154.

VAlLs, M. J. (2005) Escuchando la voz de las personas con discapacidad psíquica: Hacia una investigación cooperativa. Bordón. Revista de Pedagogía, 57 (3), 337-350.

VAN DYKe, L. (2003) Lessons in grief and death: supporting people with developmental disabilities in the healing process. Homewood, Ill., High Tide Press.

VAN LoON, J. H. M. (2009) Un sistema de apoyos centrado en la persona. Mejorando en la calidad de vida por medio de los apoyos, en Verdugo, M. A.; Nieto, T.; Jordán, B. y CRESPO, M. (coords.) Mejorando resultados personales para una calidad de vida. VII Jornadas Científicas de Investigación sobre Personas con Discapacidad. Salamanca, INICO.

Watchman, K. (2005) Practitioner-raised issues and end-of-life care for adults with Down Syndrome and dementia. Journal of Policy and Practice in Intellectual Disabilities, 2 (2), 156-162.

WATSON, L. y HARKER, M. (1993) Community care planning: a model for housing need assessment. London, Institute of Housing and National Federation of Housing Associations.

ZabalZA, M. A. y CiD, A. (2006) La tutoría en la universidad desde el punto de vista del profesorado. Bordón. Revista de Pedagogía, 58 (2), 247-267.

Zacarés, J. J. y SerRa Desfilis, E. (1997) La madurez personal: Perspectivas desde la psicología. Madrid, Pirámide. 